\title{
Nicolas LENOIR, Étude sur la Chanson d'Aiquin ou la conquête de la Bretagne par le roi Charlemagne
}

\section{Bernard Merdrignac}

\section{(2) OpenEdition}

\section{Journals}

Édition électronique

URL : http://journals.openedition.org/abpo/513

DOI : 10.4000/abpo.513

ISBN : 978-2-7535-1515-4

ISSN : 2108-6443

Éditeur

Presses universitaires de Rennes

Édition imprimée

Date de publication : 30 octobre 2009

Pagination : 211-214

ISBN : 978-2-7535-1008-1

ISSN : 0399-0826

Référence électronique

Bernard Merdrignac, "Nicolas Lenoir, Étude sur la Chanson d'Aiquin ou la conquête de la Bretagne par le roi Charlemagne ", Annales de Bretagne et des Pays de l'Ouest [En ligne], 116-3 | 2009, mis en ligne le 30 octobre 2011, consulté le 23 septembre 2020. URL : http://journals.openedition.org/abpo/513 ; DOI : https://doi.org/10.4000/abpo.513

Ce document a été généré automatiquement le 23 septembre 2020

(c) Presses universitaires de Rennes 


\title{
Nicolas LENOIR, Étude sur la Chanson d'Aiquin ou la conquête de la Bretagne par le roi Charlemagne
}

\author{
Bernard Merdrignac
}

\section{RÉFÉRENCE}

Paris, Honoré Champion, Nouvelle bibliothèque du Moyen Âge, $n^{\circ} 89$

1 Il est difficile d'imaginer un poème "à la fois plus breton et moins arthurien ", a écrit J.-C. Lozac'hmeur de la Chanson d'Aiquin. On sait que cette chanson de geste présentée par Joseph Bédier, comme le " plus ancien poème de langue française composé au delà du Coüesnon, en terre bretonne » nous est parvenue par l'intermédiaire d'une copie manuscrite $\mathrm{du}$ milieu $\mathrm{du} \mathrm{Xv}^{\mathrm{e}}$ siècle, détériorée et tronquée. Elle a fait l'objet d'appréciations divergentes de la part des critiques, du mépris de Léon Gautier ( un de nos poèmes les moins vivants et les plus médiocres ») à l'enthousiasme d'Arthur de La Borderie ( $«$ dans la grande famille des chansons de geste, celle-ci est une des meilleures et des plus intéressantes »). Le texte, publié par F. Joüon des Longrais à la fin du XIX siècle, a depuis bénéficié d'une édition critique par $\mathrm{F}$. Jacques et $\mathrm{M}$. Tyssens et d'une excellente traduction en français moderne par M. Ovazza et J.-C. Lozac'hmeur. Mais, comme l'indique en introduction Nicolas Lenoir, cette épopée qui raconte la résistance de l'archevêque de Dol Ysoré (avatar de Wicohen/Iuthowen, historiquement attesté au $\mathrm{x}^{\mathrm{e}}$ siècle) à l'invasion de la Bretagne par le chef sarrasin Aiquin et la campagne de reconquête menée par Charlemagne venu prêter main forte aux Bretons a pâtit autant de sa marginalité géographique ( la Marche de Bretagne, mais aussi la Bretagne profonde ») que de celle de ses caractères historiques : «les Vikings» (assimilés à la gent Mahon), "l'archevêque de Dol, etc.». Considérée comme "disgracieuse ou sympathique, selon le point de vue », l'œuvre a de ce fait été «marginalisée, mais non exclue, tenue en lisière du genre épique ». 
2 Aiquin, une chanson de geste marginale ? C'est d'ailleurs ainsi qu'était intitulée cette thèse de doctorat es lettres de N. Lenoir lors de sa soutenance, en 2000, devant l'Université de Rouen. Sous sa forme interrogative, ce titre rend compte du projet de la présente étude. Il s'agit de réintégrer cette chanson "plus originale que marginale » dans le corpus classique de l'épopée française. À cette fin, dans une perspective structurale, l'auteur a entrepris, pour la première fois, $d^{\prime}$ '« appréhender le poème non seulement comme une forme lyrique, mais encore comme un ensemble organisé (en vue d'une fin) de représentations et de significations, comme un système sémiotique complexe et cohérent » (p.12). La jubilation de N. Lenoir à s'attaquer à un objet d'étude «que l'empressement (mais aussi les autres priorités) de la critique » lui a permis de saisir " presque vierge de caractérisation globale » est perceptible tout au long de l'ouvrage et il a l'incontestable talent de la faire partager. Selon l'auteur, «seul J. Bédier avait tenté de mettre en rapport (mais dans un rapport dérisoire) le « fond» et la «forme » d'Aiquin. Présentée en ces termes, la question peut paraître naïve, mais articule la plupart des problématiques abordées dans ce travail»(p.675). C'est pourquoi, la démonstration de $\mathrm{N}$. Lenoir s'organise en trois parties qui permettent au lecteur d'en suivre la progression (avec intérêt et souvent avec plaisir): "Aiquin aux marges de l'histoire et de l'épopée françaises »; «les formes d'Aiquin : canons et singularités »; « le cadre épique de la guerre sainte : adoption et retournement critique ».

3 La partie centrale de l'ouvrage qui s'attache aux formes de la chanson est sans doute la plus novatrice en ce qu'elle prétend ériger ce poème en témoin d'une pratique de la technique littéraire de l'épopée française que N. Lenoir définit, en un double oxymore, comme "régulièrement originale, familièrement étrange " (p.234). L'analyse de la structure rythmico-syntaxique des vers, l'étude exhaustive de la composition des laisses (longues mais très structurées), l'inventaire des formules et des motifs stéréotypés permettent à l'auteur de faire ressortir les faiblesses, mais aussi le raffinement de l'art narratif, rhétorique et lyrique du trouvère. Le signataire du présent compte rendu n'a pas de compétence particulière pour traiter de l'emploi des outils d'analyses développés ces dernières décennies par la critique littéraire. Par contre, on peut trouver particulièrement fécondes les pistes soulevées par le caractère essentiellement lyrique de la composition qui transcende les faiblesses de l'écriture. Encore à l'orée du XIII ${ }^{e}$ siècle l'œuvre aurait tout à gagner à être envisagée sous l'angle du chant (p.238). "Aiquin gagne beaucoup à être saisie dans la perspective d'une performance orale rythmée, comme elle gagne à être replacée dans les contextes historiques et littéraires à partir desquels s'élabore sa propre architecture actantielle, narrative et politique » (p. 679).

Cette mise en perspective " civilisationniste » de l'épopée justifie l'attention portée par $\mathrm{N}$. Lenoir dans la première partie de l'ouvrage au contexte historique de la composition du poème. L'interdisciplinarité n'aurait rien à gagner d'une critique gratuite qui ferait grief à une thèse de doctorat es lettres de ne pas apporter toutes les réponses souhaitées aux questions que se posent les historiens. Le cas échéant, ceux-ci auraient plutôt intérêt à se reporter à l'étude de J.-C. Cassard "Propositions pour une lecture historique croisée du Roman d'Aiquin» (CCM, 45, 2002). Cet article, postérieur à la soutenance de la thèse de $\mathrm{N}$. Lenoir, présente le document comme « un palimpseste de l'histoire armoricaine » qui met en avant les figures de «l'archevêque de Dol et du duc Naimes » (=Nominoë) ; "une clef de lecture possible de l'œuvre» serait donc "la réticence des Bretons à accepter un pouvoir étranger » (Plantagenêt ou Capétien) à 
l'orée du XIII ${ }^{e}$ siècle La note intitulée "La Chanson d'Aiquin et saint Corentin ", mise en ligne le 29 mai 2009 par A.-Y. Bourgès sur son blog (Hagio-historiographie médiévale : [http://andreyvesbourges.blogspot.com/2009/05/la-chanson-daiquin-et-saint-corentin.html]) fournit une solide argumentation historique dont il sera fait état ci-après, mais dont il serait évidemment stupide de reprocher à l'auteur de ne pas avoir tenu compte. Ce dernier explore la piste d'un "transfert épique » à partir surtout des articles d'A. Moisan ("La Bretagne les Bretons et l'épopée française», MSHAB, 69 [1992]) et de F. Gégou ("Aiquin, personnage symbolique » Senefiance, 20 [1987] qui n'avait guère convaincu J.-C. Cassard). La figure des Plantagenêts, « oppresseurs de la Bretagne du XII ${ }^{\mathrm{e}}$ siècle» (p.14) se serait superposée à la mémoire de l'intervention en 931 dans la péninsule du chef des Normands de la Loire Incon (elle-même transposée dans le cadre de la royauté bretonne $\mathrm{du} \mathrm{IX}^{\mathrm{e}}$ siècle) «pour insinuer, sous le couvert d'une représentation féodo-vassalique conventionnelle la possibilité d'une Bretagne qui soit, comme aux grands temps, la fière égale du royaume des Francs » (p. 224). Hypothèses "un peu hardies", comme le reconnaît N. Lenoir qui concède prudemment l'éventualité d'«une interprétation moins radicale de l'idéologie autonomisante d'Aiquin » (ibid.). À ce propos, rendons au passage à P. Riché la paternité de la remarque sur les "aspirations particularistes des populations" de la Bretagne carolingienne attribuée ici (p. 38, n. 3) à J. Delumeau qui a effectivement dirigé l'Histoire de la Bretagne et des Bretons (1969), mais n'est pas le signataire du chapitre sur le haut Moyen Âge. Dans le même ordre d'idée, A. Chédeville se voit attribuer (p. 183) une formule sur les relations entre l'aristocratie franque et les chefs du regnum breton qui doit revenir à H. Guillotel, co-auteur de La Bretagne des saints et des rois (1984). Il conviendrait en outre de rectifier ici ou là certaines approximations qui affaiblissent la démonstration de l'auteur comme, par exemple (p. 209), l'inscription au nombre des « évêques de Dol » de Conwoion, le fondateur de l'abbaye Saint-Sauveur de Redon sous Nominoé! Les données archéologiques, prises en compte à juste titre dans un texte dont le «localisme" est reconnu par tous les spécialistes, gagneraient aussi à être plus rigoureusement exploitées. Ainsi, la localisation au sommet du Menez Hom du château antique dans lequel Charlemagne vient assiéger Aiquin (p. 483) fait l'impasse sur les fouilles du «camp des Salles» conduites par Ph. Guigon sur le Menez-Lokorn («la Montagne de Locronan»). En fait, la "carte 3: la Bretagne de la Chanson d'Aiquin» (p.199) illustre à elle seule les failles de l'argumentaire. La place démesurée occupée par la mythique forêt centrale implique que l'auteur demeure sous l'influence d'une interprétation romantique de l'histoire de Bretagne héritée d'A. de La Borderie et de ses émules. Dès lors, il est logique qu'il propose de dater l'œuvre (avec néanmoins toute la prudence qui s'impose) de 1196-1199 " ces années d'espérance où émerge, autour d'Arthur, Constance et Guethenoc, l'idée d'une Bretagne maîtresse de son sort » (p. 91 et n. 3). Sur un ton plus mesuré, J.-C. Cassard évoque à ce propos l'« écho assourdi du messianisme breton du XII siècle " autour d'Arthur de Bretagne (†1205) ainsi que les "signes ténus et épars » de la persistance d'une "fermentation de type "national »" après l'arrivée au pouvoir de Pierre de Dreux. A.-Y. Bourgès avance un argument décisif en faveur d'une "datation basse " (premier tiers du siècle du XIII ${ }^{\mathrm{e}}$ siècle) : le floruit de Conayn de Leon (vers 741-742), le seul membre de la famille vicomtale de Léon à avoir porté ce nom, prend place entre 1205 et 1230 . D'autre part, ce chercheur remarque que le texte dont on dispose ne peut résulter que d'une réfection tardive, postérieure à la construction, vers 1380 , de la tour Solidor à Saint-Servan qui fait l'objet d'une description précise (vers 217-229). L'éventualité de deux strates de rédaction 
(dont N. Lenoir n'écarte pas l'éventualité bien qu'il se refuse «à toute spéculation ce propos» - p. 95) ne serait-elle pas susceptible de rendre compte de l'ambiguïté de la Chanson qui renvoie dos à dos Français et Normands?

5 Le moindre mérite de cette thèse n'est pas d'outrepasser ainsi largement le niveau d'une « querelle de clocher » entre Dol et Saint-Malo à laquelle l'épopée a été souvent cantonnée à la suite de son interprétation par J. Bédier (p. 203). La dernière partie, centrée sur la mise en œuvre des stéréotypes religieux (miracles «ironiques» de Charles), approfondit l'analyse spirituelle et morale pour faire ressortir une idéologie marquée par le contemptus mundi. L'« épilogue » de cette partie, intitulé par N. Lenoir "La vanité des vanités et le mépris des empires" s'attache à l'exemplum du « renoncement d'Ahès » qui constitue une des clés de l'œuvre. Ce développement se fonde sur des «études anciennes » (les articles de F. Lot, J. Loth, G. Paris) qui « restent très intéressantes pour saisir ses origines et son sens" (p. 652). L'auteur s'en tient cependant (p. 660, n. 2) à l'opinion de F. Lot qui écartait la chanson Groah Aes (collectée par J.-M. de Penguern), considérée comme "un faux folklorique du XIXe siècle ». Cependant, la thèse de L. Pape sur La Civitas des Osismes (1978) et sa synthèse sur La Bretagne romaine (1995) ont montré tout le parti à tirer de cette chanson traditionnelle trégoroise. Celle-ci comporte des passages significatifs sur Ahès " personnification de la puissance romaine » (au même titre qu'Elen dans la tradition galloise). Le bel article de G. Milin « Le foul pencé de la femme du vieil Ohès : la folklorisation d'un apologue moral dans Aiquin» (1997) qui figure pourtant en bibliographie n'apparaît ici nulle part en note. Pourtant, sans doute aurait-il contribué à étayer l'argumentation de N. Lenoir. En "conclusion générale» (p.683), ce dernier ouvre une "perspective de lecture supplémentaire qu'on pourra qualifier avec précaution de "mythique" » et qui permettrait selon lui de donner sens «à ces fragments de l'imaginaire dit «de Bretagne » dans l'univers épique marginal d'Aiquin ». 